\title{
On some generalized invariant means and their application to the stability of the Hyers-Ulam type
}

\author{
by ROMAN BADORA (Katowice)
}

\begin{abstract}
We present some extension of the concept of an invariant mean to a space of vector-valued mappings defined on a semigroup. Next, we apply it to the study of the stability of some functional equation.
\end{abstract}

1. Introduction. In this paper, $(S, \cdot)$ denotes a semigroup, whereas $B(S, \mathbb{R})$ is the space of all real bounded functions defined on $S$.

A real linear functional $M$ on $B(S, \mathbb{R})$ is called a left (right) invariant mean if and only if

$$
\inf \{f(x): x \in S\} \leq M(f) \leq \sup \{f(x): x \in S\}, \quad f \in B(S, \mathbb{R}),
$$

and

$$
M\left({ }_{y} f\right)=M(f) \quad\left(M\left(f_{y}\right)=M(f)\right), \quad y \in S, f \in B(S, \mathbb{R}),
$$

where ${ }_{y} f$ and $f_{y}$ denote the left and right translations of $f$ defined by

$$
{ }_{y} f(x)=f(y x), \quad f_{y}(x)=f(x y), \quad x, y \in S .
$$

A mean which is simultaneously left and right invariant is said to be twosided invariant or, simply, invariant.

A semigroup $S$ which admits a left (right) invariant mean on $B(S, \mathbb{R})$ will be termed left (right) amenable. In the case where $S$ admits a twosided invariant mean on $B(S, \mathbb{R})$ we say that $S$ is two-sided amenable or just amenable.

A classical result due to J. von Neumann [17], J. Dixmier [6] and M. M. Day [3] states that every commutative semigroup is amenable, but amenability is a much weaker condition than commutativity. For the history, theory and applications of amenability of semigroups and groups see, for example, M. M. Day [4] and F. P. Greenleaf [11].

1991 Mathematics Subject Classification: 43A07, 39B70.

Key words and phrases: Hyers-Ulam stability theorem, invariant mean, binary intersection property. 
A collection $\mathcal{C}$ of sets is said to have the binary intersection property (see L. Nachbin [16] and M. M. Day [5]) if and only if every subcollection of $\mathcal{C}$, any two members of which intersect, has a non-empty intersection.

The family of all non-empty compact intervals in $\mathbb{R}$ has the binary intersection property. Furthermore, a normed space is said to have the binary intersection property if and only if the family of its closed balls has this property.

In the present paper we extend the concept of an invariant mean to some spaces of bounded vector-valued functions defined on a semigroup $S$. Z. Gajda in [9] proposed some extension of this type for the space of functions with values in a semi-reflexive space. We consider, in particular, the spaces of functions with values in spaces which have the binary intersection property.

The space of all bounded real functions on some set $A$ with the supremum norm has the binary intersection property. Obviously, this space is not semireflexive.

In the second part of this paper we present some applications of our results to an extension of the Hyers-Ulam stability theorem for homomorphisms.

2. Generalized invariant means. Now we establish a generalization of the classical Hahn-Banach theorem.

TheOREM 1. Let $X$ and $Y$ be two real linear spaces and let $\mathcal{C}$ be a translation invariant family of subsets of $Y$ having the binary intersection property. Assume that $F: X \rightarrow \mathcal{C}$ satisfies

$$
F(x+y) \subseteq F(x)+F(y), \quad x, y \in X,
$$

and

$$
F(t x)=t F(x), \quad x \in X, t \in \mathbb{R} \backslash\{0\} .
$$

Next, let $X_{0}$ be a linear subspace of $X$ and let $L_{0}: X_{0} \rightarrow Y$ be a linear operator such that $L_{0}(x) \in F(x)$ for all $x \in X_{0}$. Then there exists a linear operator $L: X \rightarrow Y$ which is an extension of $L_{0}$ and $L(x) \in F(x)$ for all $x \in X$.

This type of generalization of the Hahn-Banach theorem was considered by W. Smajdor. We omit the proof, because it is much the same as the proof of Theorem 1 in Z. Gajda, W. Smajdor and A. Smajdor [10].

We apply this theorem to the proof of some generalization of J. Dixmier's theorem (see E. Hewitt and K. A. Ross [12], Theorem 17.4). To begin with we introduce the necessary notation and terminology. 
Definition 1. Let $\mathcal{F}$ be a non-void subset of the space $Y^{S}$ of all functions from a semigroup $S$ to a set $Y$. We say that $\mathcal{F}$ is left (right) invariant if and only if $f \in \mathcal{F}$ and $y \in S$ imply that ${ }_{y} f \in \mathcal{F}\left(f_{y} \in \mathcal{F}\right)$.

Definition 2. Let $\mathcal{F}$ be a left (right) invariant linear space of functions mapping a semigroup $S$ into a linear space $Y$. Let $\mathcal{C}$ be a family of subsets of $Y$ and let $F: \mathcal{F} \rightarrow \mathcal{C}$. Then a linear operator $M: \mathcal{F} \rightarrow Y$ will be called a left (right) invariant $F$-mean if and only if

$$
M(f) \in F(f), \quad f \in \mathcal{F},
$$

and

$$
M\left({ }_{y} f\right)=M(f) \quad\left(M\left(f_{y}\right)=M(f)\right), \quad y \in S, f \in \mathcal{F} .
$$

Further, for $\mathcal{F}$ as in the above definition we denote by $\mathcal{F}_{l}\left(\mathcal{F}_{r}\right)$ the family of all functions $h: S \rightarrow Y$ of the form

$$
h=\sum_{k=1}^{n}\left(f_{k}-{ }_{y_{k}} f_{k}\right) \quad\left(h=\sum_{k=1}^{n}\left(f_{k}-\left(f_{k}\right)_{y_{k}}\right)\right),
$$

where $f_{k} \in \mathcal{F}$ and $y_{k} \in S$ for $k=1, \ldots, n$.

Now, the theorem of J. Dixmier can be reformulated in the following way:

THeOREm 2. Let $\mathcal{F}$ be a left (right) invariant linear space of functions defined on a semigroup $S$ with values in a real linear space $Y$. Let $\mathcal{C}$ be a translation invariant family of subsets of $Y$ having the binary intersection property and let $F: \mathcal{F} \rightarrow \mathcal{C}$ satisfy

$$
F(f+g) \subseteq F(f)+F(g), \quad f, g \in \mathcal{F},
$$

and

$$
F(t f)=t F(f), f \in \mathcal{F}, \quad t \in \mathbb{R} \backslash\{0\} .
$$

Then there exists a left (right) invariant $F$-mean on $\mathcal{F}$ if and only if

$$
0 \in F(h)
$$

for all functions $h$ from the family $\mathcal{F}_{l}\left(\mathcal{F}_{r}\right)$.

The above result yields a generalizaton of J. Dixmier's theorem which we obtain by taking for $\mathcal{C}$ the family of all non-empty compact convex subsets of $\mathbb{R}$ and putting

$$
F(f)=\left[\inf _{x \in S} f(x), \sup _{x \in S} f(x)\right], \quad f \in \mathcal{F},
$$

where $\mathcal{F}$ is a left (right) invariant linear subspace of the space of all bounded real functions on $S$. 
Pro of of Theorem 2. Let $M$ be a left invariant $F$-mean on the space $\mathcal{F}$ and fix $h=\sum_{k=1}^{n}\left(f_{k}-y_{k} f_{k}\right)$ with $f_{k} \in \mathcal{F}$ and $y_{k} \in S(k=1, \ldots, n)$. Then

$$
M(h)=M\left(\sum_{k=1}^{n}\left(f_{k}-{ }_{y_{k}} f_{k}\right)\right)=\sum_{k=1}^{n}\left(M\left(f_{k}\right)-M\left(f_{k}\right)\right)=0
$$

and so $0=M(h) \in F(h)$.

Conversely, if $0 \in F(h)$ for each $h \in \mathcal{F}_{l}$, then let $M_{0}: \mathcal{F}_{l} \rightarrow Y$ be the zero operator. Since $M_{0}(h) \in F(h)$ for $h \in \mathcal{F}_{l}$, Theorem 1 implies that there exists a linear operator $M: \mathcal{F} \rightarrow Y$ such that

$$
M(f) \in F(f), \quad f \in \mathcal{F}, \quad \text { and } \quad M(h)=M_{0}(h)=0, \quad h \in \mathcal{F}_{l} .
$$

Then $M$ is a left invariant $F$-mean.

The proof of the "right invariant" version is analogous.

Let $Y$ be a linear topological space. A subset $A$ of $Y$ is called bounded if and only if it is absorbed by any neighbourhood of zero in the topology of $Y$. The space of all bounded functions (i.e. those with bounded image) from a semigroup $S$ into $Y$ will be denoted by $B(S, Y)$.

THEOREM 3. Let $(S, \cdot)$ be a left (right) amenable semigroup and let $Y$ be a real locally convex space. Let $\mathcal{F}$ be a left (right) invariant linear subspace of $B(S, Y)$. Let $\mathcal{C}$ be a translation invariant collection of closed convex sets in $Y$ having the binary intersection property. Assume that the map $F: \mathcal{F} \rightarrow \mathcal{C}$ satisfies (9), (10) and

$$
h(S) \subseteq F(h), \quad h \in \mathcal{F}_{l}\left(h \in \mathcal{F}_{r}\right) .
$$

Then there exists a left (right) invariant $F$-mean on the space $\mathcal{F}$.

Pro of. We restrict ourselves to the "left invariant" version.

Clearly, by Theorem 2 it is enough to show that $0 \in F(h)$ for every $h \in \mathcal{F}_{l}$. Suppose that there exists an $h \in \mathcal{F}_{l}$ such that

$$
0 \notin F(h),
$$

and let $h=\sum_{k=1}^{n}\left(f_{k}-y_{k} f_{k}\right)$, for some $f_{k} \in \mathcal{F}$ and $y_{k} \in S(k=1, \ldots, n)$. Applying to the sets $\{0\}$ and $F(h)$ the separation theorem in a locally convex space (see e.g. M. M. Day [5], Theorem 5, p. 24) we obtain the existence of a continuous linear functional $L$ on $Y$ such that

$$
0=L(0)>\sup _{y \in F(h)} L(y) .
$$

For $k=1, \ldots, n$ let us define bounded real functions $\widetilde{f}_{k}$ on $S$ by

$$
\widetilde{f}_{k}(x)=L\left(f_{k}(x)\right), \quad x \in S,
$$


and let $M$ be a left invariant mean on $B(S, \mathbb{R})$, which exists by our assumption. Then

$$
0=M\left(\sum_{k=1}^{n}\left(\widetilde{f}_{k}-{ }_{y_{k}} \widetilde{f}_{k}\right)\right) \leq \sup _{x \in S} \sum_{k=1}^{n}\left(\widetilde{f}_{k}(x)-{ }_{y_{k}} \widetilde{f}_{k}(x)\right)
$$

and, on the other hand,

$$
\begin{aligned}
\sup _{x \in S} \sum_{k=1}^{n}\left(\widetilde{f}_{k}(x)-{ }_{y_{k}} \widetilde{f}_{k}(x)\right) & =\sup _{x \in S} \sum_{k=1}^{n}\left(L\left(f_{k}(x)\right)-L\left(y_{k} f_{k}(x)\right)\right) \\
& =\sup _{x \in S} L(h(x))=\sup _{y \in h(S)} L(y) \leq \sup _{y \in F(h)} L(y)<0,
\end{aligned}
$$

which is impossible. Therefore, condition (11) is satisfied.

The utility of Theorem 3 depends on the existence of non-trivial examples of families $\mathcal{C}$ and maps $F$. In the present note we concentrate our attention on the space of all bounded functions defined on a semigroup $S$ and taking values in a normed space $Y$ which has the binary intersection property.

We start with the following lemmas:

LEMMA 1. If the family $\mathcal{C}$ has the binary intersection property, then the family $\widetilde{\mathcal{C}}$ of all non-empty intersections of subfamilies of $\mathcal{C}$ also has the binary intersection property.

Proof. Let $\widetilde{\mathcal{C}_{1}}=\left\{A_{q}: q \in Q\right\}$ be a subfamily of $\widetilde{\mathcal{C}}$ any two members of which intersect. Then for each $q \in Q$ we have

$$
A_{q}=\bigcap\left\{B_{i}^{q}: i \in I_{q}\right\}
$$

for some subfamily $\left\{B_{i}^{q}: i \in I_{q}\right\}$ of $\mathcal{C}$ and any two members of the family $\mathcal{C}_{1}=\left\{B_{i}^{q}: i \in I_{q}, q \in Q\right\}$ intersect. Since $\mathcal{C}$ has the binary intersection property, $\mathcal{C}_{1}$ has non-empty intersection. Therefore, the intersection of $\widetilde{\mathcal{C}}_{1}$, which coincides with the intersection of $\mathcal{C}_{1}$, is non-empty.

The following lemma is some version of the results obtained by K. Nikodem in [18] and by Z. Gajda, W. Smajdor and A. Smajdor in [10].

LEMMA 2. Let $\mathcal{C}$ be a translation invariant family of subsets of the linear space $Y$ having the binary intersection property. Suppose that $\mathcal{C}$ is also invariant with respect to symmetry about zero. If $\left\{A_{i}: i \in I\right\}$ and $\left\{B_{q}: q \in Q\right\}$ are two subfamilies of $\mathcal{C}$ such that

$$
\bigcap\left\{A_{i}: i \in I\right\} \neq \emptyset \text { and } \bigcap\left\{B_{q}: q \in Q\right\} \neq \emptyset,
$$

then

$$
\bigcap\left\{A_{i}+B_{q}: i \in I, q \in Q\right\}=\bigcap\left\{A_{i}: i \in I\right\}+\bigcap\left\{B_{q}: q \in Q\right\} .
$$


Proof. The inclusion $\supseteq$ is obvious. To prove the inverse inclusion it is enough to show that

$$
\bigcap\left\{U_{i}+W: i \in I\right\} \subseteq \bigcap\left\{U_{i}: i \in I\right\}+W
$$

for $\left\{U_{i}: i \in I\right\} \subseteq \widetilde{\mathcal{C}}$ such that $\bigcap\left\{U_{i}: i \in I\right\} \neq \emptyset$ and $W \in \widetilde{\mathcal{C}}$, where $\widetilde{\mathcal{C}}$ denotes the family of all non-empty intersections of subfamilies of $\mathcal{C}$.

Take $v \in \bigcap\left\{U_{i}+W: i \in I\right\}$. Then for each $i \in I$ there exist $u_{i} \in U_{i}$ and $w_{i} \in W$ such that $v=u_{i}+w_{i}$. Hence $U_{i} \cap(v-W) \neq \emptyset$ for all $i \in I$. Since $\left\{U_{i}: i \in I\right\} \cup\{v-W\}$ is a subfamily of $\widetilde{\mathcal{C}}$ having the binary intersection property and any two members of this subfamily intersect, we have

$$
\bigcap\left\{U_{i}: i \in I\right\} \cap(v-W) \neq \emptyset .
$$

Let $u \in \bigcap\left\{U_{i}: i \in I\right\} \cap(v-W)$. Then

$$
v=u+(v-u) \in \bigcap\left\{U_{i}: i \in I\right\}+W .
$$

Now, let $(S, \cdot)$ be a semigroup and let $(Y,\|\cdot\|)$ be a normed space which has the binary intersection property. Under these assumptions we will prove the following auxiliary result.

LEMMA 3. Let $\mathcal{C}_{b}$ be the family of all non-empty intersections of closed balls in $Y$. Then the map $F_{b}: B(S, Y) \rightarrow \mathcal{C}_{b}$ defined by

$$
F_{b}(f)=\bigcap\{B: B \text { is a closed ball in } Y \text { and } f(S) \subseteq B\}, \quad f \in B(S, Y) \text {, }
$$

satisfies conditions (9), (10) and (12).

In the proof we will use the following fact:

Remark 1. Let $(Y,\|\cdot\|)$ be a normed space. Then for every $y, \widetilde{y} \in Y$ and $r, \widetilde{r}>0$, one has

$$
b(y, r)+b(\widetilde{y}, \widetilde{r})=b(y+\widetilde{y}, r+\widetilde{r}),
$$

where $b(y, r)$ denotes the closed ball in $Y$ with centre at $y$ and radius $r$.

Pr o of. The inclusion $\subseteq$ is immediate. To prove the inverse inclusion, fix $z \in b(y+\widetilde{y}, r+\widetilde{r})$. Then $z=u+v$, where

$$
u=\frac{r}{r+\widetilde{r}}(z-y-\widetilde{y})+y \quad \text { and } \quad v=\frac{\widetilde{r}}{r+\widetilde{r}}(z-y-\widetilde{y})+\widetilde{y} .
$$

Moreover,

and

$$
\|u-y\|=\frac{r}{r+\widetilde{r}}\|z-y-\widetilde{y}\| \leq r
$$

$$
\|v-\widetilde{y}\|=\frac{\widetilde{r}}{r+\widetilde{r}}\|z-y-\widetilde{y}\| \leq \widetilde{r} .
$$

Therefore, $z \in b(y, r)+b(\widetilde{y}, \widetilde{r})$. 
Proof of Lemma 3. Fix $f, g \in B(S, Y)$. Then for any closed balls $B$ and $\widetilde{B}$ such that $f(S) \subseteq B$ and $g(S) \subseteq \widetilde{B}$ we have

$$
(f+g)(S) \subseteq f(S)+g(S) \subseteq B+\widetilde{B}
$$

and $B+\widetilde{B}$ is a closed ball in $Y$. Hence,

$$
F_{b}(f+g) \subseteq B+\widetilde{B} .
$$

By Lemma 2 this implies (9).

To prove (10) we fix $f \in B(S, Y)$ and $t \in \mathbb{R} \backslash\{0\}$. Then

$$
\begin{aligned}
t F_{b}(f) & =t \bigcap\{B: B \text { is a closed ball in } Y \text { and } f(S) \subseteq B\} \\
& =\bigcap\left\{t B: B \text { is a closed ball in } Y \text { and } f(S) \subseteq t^{-1} t B\right\} \\
& =\bigcap\{B: B \text { is a closed ball in } Y \text { and } t f(S) \subseteq B\}=F_{b}(t f) .
\end{aligned}
$$

Evidently, the map $F_{b}$ also satisfies (12).

From Theorem 3 we obtain:

Theorem 4. Let $(S, \cdot)$ be a left (right) amenable semigroup and let $(Y,\|\cdot\|)$ be a real normed space having the binary intersection property. Then there exists a linear operator $M: B(S, Y) \rightarrow Y$ such that

$$
M(f) \in b(f), \quad f \in B(S, Y),
$$

and

$$
M\left({ }_{y} f\right)=M(f) \quad\left(M\left(f_{y}\right)=M(f)\right), \quad f \in B(S, Y), y \in S,
$$

where $b(f)$ denotes the intersection of all closed balls in $Y$ including $f(S)$.

3. An application. In what follows we assume that $(S, \cdot)$ is a semigroup and $(Y,\|\cdot\|)$ is a real normed space. A function $f: S \rightarrow Y$ is said to be additive provided it satisfies the Cauchy functional equation

$$
f(x y)=f(x)+f(y), \quad x, y \in S .
$$

Further, $f: S \rightarrow Y$ is called approximately additive if and only if there is an $\varepsilon \geq 0$ such that

$$
\|f(x y)-f(x)-f(y)\| \leq \varepsilon
$$

for all $x, y \in S$. For $f: S \rightarrow Y$ and $x$ and $y$ in $S$ the difference

$$
f(x y)-f(x)-f(y)
$$

is called the Cauchy difference and denoted by $C_{(x, y)} f$.

S. M. Ulam [22] raised the following problem. Suppose that a function $f$ from a Banach space $X$ into another Banach space $Y$ satisfies (18). Does 
there exist an additive function $a: X \rightarrow Y$ and a $k>0$ such that

$$
\|f(x)-a(x)\| \leq k \varepsilon, \quad x \in X ?
$$

A solution to this problem (with $k=1$ ) was given in 1941 by D. H. Hyers [13]. Later his result was carried over to a higher level of abstraction by J. Rätz [19] and Z. Moszner [15]. For our discussion the main motivation is a result of L. Székelyhidi (see [20] and [21]) who pointed out that the classical Hyers theorem holds true for functions mapping an amenable semigroup into a reflexive space.

With the aid of Theorem 4, using the method of the proof of Székelyhidi's theorem, one may prove the following

Theorem 5. Let $(S, \cdot)$ be a left (right) amenable semigroup and let $(Y,\|\cdot\|)$ be a real normed space having the binary intersection property. If $f: S \rightarrow Y$ satisfies (18) for some $\varepsilon \geq 0$, then there exists a unique additive function $a: S \rightarrow Y$ such that

$$
\|f(x)-a(x)\| \leq \varepsilon, \quad x \in S .
$$

As a consequence we get the completeness of any real normed space having the binary intersection property. In fact, this is an immediate conclusion from Theorem 5 and a result of G. L. Forti and J. Schwaiger (see [7], Theorem 7).

M. A. Albert and J. A. Baker in [1] proved that if $(S,+)$ is a commutative semigroup and a mapping $F$ from the Cartesian product $S^{n}(n \in \mathbb{N})$ into some Banach space $Y$ has bounded Cauchy difference with respect to each variable separately, then there exists an $n$-additive function $A: S^{n} \rightarrow Y$ such that $F-A$ is bounded.

Now we consider another problem of this type, which was suggested to me by Zbigniew Gajda. Let $(S, \cdot)$ and $(\widetilde{S}, \cdot)$ be semigroups, let $Y$ be a real linear space and let $H: S \times \widetilde{S} \rightarrow Y$. Given $x, y \in S$ and $u, v \in \widetilde{S}$ we consider the Cauchy difference operators $C_{(x, y)}^{1}$ and $C_{(u, v)}^{2}$ acting on $H$ regarded as a function of the first or the second variable, respectively. We will study the stability and solutions of the functional equation

$$
C_{(u, v)}^{2} C_{(x, y)}^{1} H=0
$$

for all $x, y \in S$ and $u, v \in \widetilde{S}$, i.e.

$$
\begin{aligned}
& H(x y, u v)-H(x, u v)-H(y, u v) \\
& -H(x y, u)+H(x, u)+H(y, u) \\
& -H(x y, v)+H(x, v)+H(y, v)=0
\end{aligned}
$$

for all $x, y \in S$ and $u, v \in \widetilde{S}$.

It is easy to check that if $A, B: S \times \widetilde{S} \rightarrow Y$ are such that $A(\cdot, u): S \rightarrow Y$ is additive for any fixed $u \in \widetilde{S}$ and $B(x, \cdot): \widetilde{S} \rightarrow Y$ is additive for any fixed 
$x \in S$, then $H: S \times \widetilde{S} \rightarrow Y$ defined by

$$
H=A+B
$$

satisfies equation (20). Therefore, our study is similar to that of Z. Gajda in [8] (for polynomial functions).

THEOREM 6. Let $(S, \cdot)$ and $(\widetilde{S}, \cdot)$ be left (right) amenable semigroups, let $(Y,\|\cdot\|)$ be a real normed space having the binary intersection property and let $\varepsilon \geq 0$. Assume that $F: S \times \widetilde{S} \rightarrow Y$ satisfies

$$
\left\|C_{(u, v)}^{2} C_{(x, y)}^{1} F\right\| \leq \varepsilon
$$

for all $x, y \in S$ and $u, v \in \widetilde{S}$. Then there exists a unique map $H: S \times \widetilde{S} \rightarrow Y$ of the form (21) which satisfies equation (20) and such that

$$
\|F(x, u)-H(x, u)\| \leq \varepsilon
$$

for all $x \in S$ and all $u \in \widetilde{S}$.

Proof. We only prove the "left version".

Condition (22) implies that the mapping $g$ defined by

$$
S \times S \ni(x, y) \mapsto F(x y, \cdot)-F(x, \cdot)-F(y, \cdot)
$$

assumes values in the space $A A(\widetilde{S}, Y)$ of all functions from $\widetilde{S}$ into $Y$ which have bounded Cauchy difference.

If $f \in A A(\widetilde{S}, Y)$ then there exists a constant $k \geq 0$ such that

$$
\left\|C_{(u, v)} f\right\|=\|f(u v)-f(u)-f(v)\| \leq k
$$

for all $u, v \in \widetilde{S}$. Therefore, from Theorem 5 it follows that there exists a unique additive function $a_{f}: \widetilde{S} \rightarrow Y$ such that the map $r_{f}=f-a_{f}$ is bounded (by $k$ ) on $\widetilde{S}$.

Let $A(\widetilde{S}, Y)$ denote the linear subspace of $A A(\widetilde{S}, Y)$ consisting of all additive functions and let $\widetilde{Y}$ stand for the quotient space $A A(\widetilde{S}, Y) / A(\widetilde{S}, Y)$. Every element of $\widetilde{Y}$ can be uniquely represented in the form $r+A(\widetilde{S}, Y)$, where $r \in A A(\widetilde{S}, Y)$ is bounded, i.e. $r \in B(\widetilde{S}, Y)$.

We define a norm on $\widetilde{Y}$ by

$$
\|r+A(\widetilde{S}, Y)\|=\sup \{\|r(u)\|: u \in \widetilde{S}\}
$$

for all $r \in B(\widetilde{S}, Y)$.

Since the space $Y$ has the binary intersection property it is evident that so do $B(\widetilde{S}, Y)$ and $\widetilde{Y}$. Moreover, for any $f \in A A(\widetilde{S}, Y)$ the norm of $f+$ $A(\widetilde{S}, Y)$ in $\widetilde{Y}$ is not larger than the constant which bounds the Cauchy difference of $f$. 
Theorem 4 implies that there exists a linear operator $M: B(S, \widetilde{Y}) \rightarrow \widetilde{Y}$ such that

$$
M(g) \in b(g), \quad g \in B(S, \tilde{Y})
$$

and

$$
M\left({ }_{z} g\right)=M(g), \quad z \in S, g \in B(S, \widetilde{Y}) .
$$

Now we return to the map $g$ defined by (24). Fix $x \in S$. Then the mapping $G(x, \cdot): S \rightarrow \widetilde{Y}$ defined by

$$
G(x, y)=g(x, y)+A(\widetilde{S}, Y), \quad y \in S,
$$

is bounded (in norm) by $\varepsilon$, i.e. $G(x, \cdot) \in B(S, \widetilde{Y})$ for each $x \in S$. Let

$$
h(x)=M_{y}(G(x, y)), \quad x \in S,
$$

where the subscript $y$ indicates that $M$ is applied to a function of the variable $y$. Then for any $x \in S$,

$$
h(x) \in b_{\widetilde{Y}}(0, \varepsilon),
$$

where $b_{\widetilde{Y}}(0, \varepsilon)$ denotes the closed ball in $(\widetilde{Y},\|\cdot\|)$ with centre at zero and radius $\varepsilon$ (if $\varepsilon=0$ then we put $b_{\widetilde{Y}}(0,0)=\{0\}$ ).

Therefore, for each $x \in S$ there exists a (unique) function $r(x): \widetilde{S} \rightarrow Y$ such that

$$
h(x)=r(x)+A(\widetilde{S}, Y)
$$

and

$$
\|r(x)(u)\| \leq \varepsilon, \quad u \in \widetilde{S}
$$

Moreover,

$$
h(x \widetilde{x})=h(x)+h(\widetilde{x})-G(x, \widetilde{x}), \quad x, \widetilde{x} \in S .
$$

Indeed, for any $x, \widetilde{x} \in S$, from the left invariance of $M$, we get

$$
\begin{aligned}
h(x)+h(\widetilde{x})-G(x, \widetilde{x})= & M_{y}(F(x y, \cdot)-F(x, \cdot)-F(y, \cdot)+A(\widetilde{S}, Y)) \\
& +M_{y}(F(\widetilde{x} y, \cdot)-F(\widetilde{x}, \cdot)-F(y, \cdot)+A(\widetilde{S}, Y)) \\
& -[F(x \widetilde{x}, \cdot)-F(x, \cdot)-F(\widetilde{x}, \cdot)+A(\widetilde{S}, Y)] \\
= & M_{y}(F(x \widetilde{x} y, \cdot)-F(x, \cdot)-F(\widetilde{x} y, \cdot)+A(\widetilde{S}, Y)) \\
& +M_{y}(F(\widetilde{x} y, \cdot)-F(\widetilde{x}, \cdot)-F(y, \cdot)+A(\widetilde{S}, Y)) \\
& -[F(x \widetilde{x}, \cdot)-F(x, \cdot)-F(\widetilde{x}, \cdot)+A(\widetilde{S}, Y)] \\
= & M_{y}(F(x \widetilde{x} y, \cdot)-F(x \widetilde{x}, \cdot)-F(y, \cdot)+A(\widetilde{S}, Y)) \\
= & M_{y}(G(x \widetilde{x}, y))=h(x \widetilde{x}) .
\end{aligned}
$$


This means that

$$
F(x \widetilde{x}, \cdot)+r(x \widetilde{x})-F(x, \cdot)-r(x)-F(\widetilde{x}, \cdot)-r(\widetilde{x}) \in A(\widetilde{S}, Y)
$$

for all $x, \widetilde{x} \in S$.

Moreover, condition (31) states that the Cauchy difference of the function

$$
S \ni x \mapsto F(x, \cdot)+r(x)
$$

is in the linear subspace $A(\widetilde{S}, Y)$ of $Y^{\widetilde{S}}$. Therefore, the theorem of $\mathrm{K}$. Baron [2] (see also Z. Gajda [8], Lemma 1) guarantees the existence of an additive function $a$ from $S$ into $Y^{\widetilde{S}}$ such that

$$
F(x, \cdot)+r(x)-a(x) \in A(\widetilde{S}, Y), \quad x \in S .
$$

Hence, for every $x \in S$, there exists an additive function $B(x, \cdot): \widetilde{S} \rightarrow Y$ such that

$$
F(x, u)+r(x)(u)-a(x)(u)=B(x, u), \quad u \in \widetilde{S} .
$$

Now, let us put

$$
A(x, u)=a(x)(u), \quad x \in S, u \in \widetilde{S} .
$$

Then $A(\cdot, u): S \rightarrow Y$ is additive for each $u \in \widetilde{S}$ and

$$
F(x, u)-A(x, u)-B(x, u)=-r(x)(u), \quad x \in S, u \in \widetilde{S} .
$$

Therefore, the mapping $H: S \times \widetilde{S} \rightarrow Y$ defined by

$$
H(x, u)=A(x, u)+B(x, u), \quad x \in S, u \in \widetilde{S},
$$

is of the form (21) and moreover, by (29),

$$
\|F(x, u)-H(x, u)\|=\|r(x)(u)\| \leq \varepsilon
$$

for all $x \in S$ and all $u \in \widetilde{S}$, which ends the existence part of the proof.

To prove uniqueness, suppose that $H, \widetilde{H}: S \times \widetilde{S} \rightarrow Y$ satisfy equation (20) and inequality (23). Then from (20), by induction, we have

$$
H\left(x^{2^{n}}, u^{2^{n}}\right)=2^{n} H\left(x^{2^{n}}, u\right)+2^{n} H\left(x, u^{2^{n}}\right)-4^{n} H(x, u)
$$

and

$$
\widetilde{H}\left(x^{2^{n}}, u^{2^{n}}\right)=2^{n} \widetilde{H}\left(x^{2^{n}}, u\right)+2^{n} \widetilde{H}\left(x, u^{2^{n}}\right)-4^{n} \widetilde{H}(x, u)
$$

for all $x \in S, u \in \widetilde{S}, n \in \mathbb{N}$. By (23) we get 


$$
\begin{aligned}
\| H(x, u)- & \widetilde{H}(x, u) \| \\
= & 4^{-n} \| H\left(x^{2^{n}}, u^{2^{n}}\right)-2^{n} H\left(x^{2^{n}}, u\right)-2^{n} H\left(x, u^{2^{n}}\right) \\
& \quad-\widetilde{H}\left(x^{2^{n}}, u^{2^{n}}\right)+2^{n} \widetilde{H}\left(x^{2^{n}}, u\right)+2^{n} \widetilde{H}\left(x, u^{2^{n}}\right) \| \\
\leq & 4^{-n}\left\{\left\|H\left(x^{2^{n}}, u^{2^{n}}\right)-F\left(x^{2^{n}}, u^{2^{n}}\right)\right\|+\left\|F\left(x^{2^{n}}, u^{2^{n}}\right)-\widetilde{H}\left(x^{2^{n}}, u^{2^{n}}\right)\right\|\right. \\
& +2^{n}\left\|H\left(x^{2^{n}}, u\right)-F\left(x^{2^{n}}, u\right)\right\|+2^{n}\left\|F\left(x^{2^{n}}, u\right)-\widetilde{H}\left(x^{2^{n}}, u\right)\right\| \\
& \left.+2^{n}\left\|H\left(x, u^{2^{n}}\right)-F\left(x, u^{2^{n}}\right)\right\|+2^{n}\left\|F\left(x, u^{2^{n}}\right)-\widetilde{H}\left(x, u^{2^{n}}\right)\right\|\right\} \\
\leq & 4^{-n}\left\{2 \varepsilon+2^{n} 2 \varepsilon+2^{n} 2 \varepsilon\right\} \underset{n \rightarrow \infty}{\longrightarrow} 0,
\end{aligned}
$$

which yields $H=\widetilde{H}$.

This theorem states that equation (20) is stable on amenable semigroups and moreover (with $\varepsilon=0$ ) that any solution of this equation is of the form (21). This leads to the following

TheOrem 7. Let $(S, \cdot)$ and $(\widetilde{S}, \cdot)$ be left (right) amenable semigroups, let $(Y,\|\cdot\|)$ be a real normed space having the binary intersection property and let $H: S \times \widetilde{S} \rightarrow Y$ satisfy equation (20). Then $H$ is of the form (21).

\section{References}

[1] M. A. Albert and J. A. Baker, Functions with bounded n-th differences, Ann. Polon. Math. 43 (1983), 93-103.

[2] K. Baron, Functions with differences in subspaces, in: Proceedings of the 18th International Symposium on Functional Equations, University of Waterloo, Faculty of Mathematics, Waterloo, Ontario, Canada, 1980.

[3] M. M. Day, Amenable semigroups, Illinois J. Math. 1 (1957), 509-544.

[4] -, Fixed point theorem for compact convex sets, ibid. 5 (1961), 585-590.

[5] —, Normed Linear Spaces, Springer, Berlin 1973.

[6] J. Dixmier, Les moyennes invariantes dans les semigroupes et leurs applications, Acta Sci. Math. (Szeged) 12 (1950), 213-227.

[7] G. L. Forti and J. Schwaiger, Stability of homomorphisms and completeness, C. R. Math. Rep. Acad. Sci. Canada 11 (6) (1989), 215-220.

[8] Z. Gajda, A solution to a problem of J. Schwaiger, Aequationes Math. 32 (1987), $38-44$.

[9] - Invariant means and representations of semigroups in the theory of functional equations, Prace Naukowe Uniwersytetu Śląskiego 1273, Katowice 1992.

[10] Z. Gajda, W. Smajdor and A. Smajdor, A theorem of the Hahn-Banach type and its applications, Ann. Polon. Math. 57 (1992), 243-252.

[11] F. P. Greenleaf, Invariant Means on Topological Groups and Their Applications, Van Nostrand Math. Stud. 16, New York 1969.

[12] E. Hewitt and K. A. Ross, Abstract Harmonic Analysis, Vol. I, Springer, Berlin 1963. 
[13] D. H. Hyers, On the stability of the linear functional equations, Proc. Nat. Acad. Sci. U.S.A. 27 (1941), 222-224.

[14] M. Kuczma, An Introduction to the Theory of Functional Equations and Inequalities, Polish Scientific Publishers (PWN) and Silesian University Press, WarszawaKraków-Katowice 1985.

[15] Z. Moszner, Sur la stabilité de l'équation d'homomorphisme, Aequationes Math. 29 (1985), 290-306.

[16] L. Nachbin, A theorem of the Hahn-Banach type for linear transformations, Trans. Amer. Math. Soc. 68 (1950), 28-46.

[17] J. von Neumann, Zur allgemeinen Theorie der Masses, Fund. Math. 13 (1929), 73-116.

[18] K. Nikodem, On Jensen's functional equation for set-valued functions, Rad. Mat. 3 (1987), 23-33.

[19] J. Rätz, On approximately additive mappings, in: General Inequalities 2, Internat. Ser. Numer. Math. 47, Birkhäuser, Basel 1980, 233-251.

[20] L. Székelyhidi, Remark 17, Report of Meeting, Aequationes Math. 29 (1985), 95-96.

[21] —, Note on Hyers's theorem, C. R. Math. Rep. Acad. Sci. Canada 8 (1986), 127-129.

[22] S. M. Ulam, Problems in Modern Mathematics, Science Editions, Wiley, New York 1960.

INSTITUTE OF MATHEMATICS

SILESIAN UNIVERSITY

BANKOWA 14

40-007 KATOWICE, POLAND 